\title{
Input-output implementation of the Youla architecture
}

\author{
Niemann, Hans Henrik
}

Published in:

Proceedings of 2021 American Control Conference

Publication date:

2021

Document Version

Publisher's PDF, also known as Version of record

Link back to DTU Orbit

Citation $(A P A)$ :

Niemann, H. H. (2021). Input-output implementation of the Youla architecture. In Proceedings of 2021 American Control Conference

\section{General rights}

Copyright and moral rights for the publications made accessible in the public portal are retained by the authors and/or other copyright owners and it is a condition of accessing publications that users recognise and abide by the legal requirements associated with these rights.

- Users may download and print one copy of any publication from the public portal for the purpose of private study or research.

- You may not further distribute the material or use it for any profit-making activity or commercial gain

- You may freely distribute the URL identifying the publication in the public portal

If you believe that this document breaches copyright please contact us providing details, and we will remove access to the work immediately and investigate your claim 


\title{
Input-output implementation of the Youla architecture
}

\author{
Henrik Niemann
}

\begin{abstract}
The well-known controller architecture based on the Youla parameterization is revisited in this paper. The key result in this paper is a reformulation of the Youla controller such that an exact implementation of the Youla parameterization part can be done using only terminals of the nominal controller. Further, the parameterization part does not use the nominal feedback controller directly.

Keyword:

Youla controller architecture, controller parameterization, coprime factorization.
\end{abstract}

\section{INTRODUCTION}

The Youla controller architecture has been central in control theory since it was developed more than forty years ago by Youla et.al, [20], [21] and by Kucera, [5]. Further, the elegant state-space description of the coprime factors by Nett et.al, [9] is one of the developments that simplifies the application of the Youla parameterization. In following years, the Youla parameterization had been applied in connection with different areas as e.g. system identification, [1], [12], optimization, [2], [3], performance optimization, [18], [23] as well as fault diagnosis and fault-tolerant control, [10], [13].

The standard implementation of the Youla parameterization based on coprime factorization as described in e.g. [18], [23] requires an exact knowledge of both the system as well as the applied feedback controller. Another important issue is that it requires access to internal signals in the controller. This might not always be possible in existing controllers. As a consequence of this, it might not be possible to apply the standard Youla architecture directly to existing controllers.

There exist controller architectures where the parameterization part is only connected to the nominal controller through its terminals. The internal model control (IMC) for stable systems is well known and applied, see e.g. [2], [8]. IMC part of the controllers is required to be stable will result in a stable closed-loop system. This is equivalent to the condition for the Youla matrix transfer function. If the system is open-loop unstable, the IMC controller cannot be applied. Instead, the architecture described in [15] can be applied. Here, a model of the closed-loop system is applied and the difference between the real closed-loop system and the model of it is applied for the input to the free stable Youla matrix transfer function.

A more direct approach based on the Youla parameterization is described in [17]. In this architecture, the feedback part from the Youla matrix transfer function is

H. Niemann is with Dept. of Electrical Engineering, Automation and Control, Build. 326, Technical University of Denmark, DK-2800 Kgs. Lyngby, Denmark, hhn@elektro.dtu.dk only connected to the controller output terminal. For stable controllers, the Youla matrix transfer function can be selected such that the original architecture is obtained. For unstable controllers, it will require an unstable Youla matrix transfer function for obtaining this. The right half plane (RHP) pole structure from the nominal controller needs to be included in the Youla matrix transfer function. This controller architecture has been applied in [6].

It should also be mentioned that there exist alternative methods to the Youla parameterization. A system-level parameterization has been described in [19]. An equivalent input-output parameterization is described in [4]. These two parameterizations are not based on a coprime factorization of the nominal system and controller as well as on the Bezout equation. In [22], it has been shown that the Youla, the system-level, and the input-output parameterization are equivalent. It is shown how it is possible to transform the controllers between the three different architectures.

The main result in this paper is a reformulation of the original Youla architecture so it can be used in connection with existing controllers without using any internal signals. It is shown that the original Youla architecture can be reformulated such that the parameterization part is an addon part on the nominal feedback controller. It is possible to substitute internal control signals exactly by using the controller terminals. The basis for this substitution is the use of the Bezout equation together with coprime factors for the system. An important issue in connection with this implementation of the Youla architecture is that the parameterization part is independent of the nominal feedback controller. The new architecture gives the possibility to apply the parameterization part as an add-on part to existing unknown controllers. This will allow to use learning-based or data-driven methods for the design and optimization of the Youla matrix transfer function.

The rest of this paper is organized as follows. The system set-up and some preliminary results are given in Section II. The main result of the paper is given in Section III. An example is given in Section IV and the paper is closed with a short conclusion in Section V.

\section{System Setup and Preliminary Results}

Let a general system be given by:

$$
\Sigma:\left\{\left(\begin{array}{l}
z \\
y
\end{array}\right)=\left(\begin{array}{ll}
G_{z w} & G_{z u} \\
G_{y w} & G_{y u}
\end{array}\right)\left(\begin{array}{l}
w \\
u
\end{array}\right)\right.
$$

where $w \in \mathcal{R}^{k}$ is an external input vector, $u \in \mathcal{R}^{m}$ is the control input signal vector, $z \in \mathcal{R}^{k}$ is an external output vector, and $y \in \mathcal{R}^{p}$ is the measurement vector. 
A state space description of (1) is given by:

$$
\Sigma:\left\{\left(\begin{array}{c}
\dot{x} \\
z \\
y
\end{array}\right)=\left(\begin{array}{c|cc}
A & B_{w} & B_{u} \\
\hline C_{z} & D_{z w} & D_{z u} \\
C_{y} & D_{y w} & D_{y u}
\end{array}\right)\left(\begin{array}{c}
x \\
w \\
u
\end{array}\right)\right.
$$

where $x \in \mathcal{R}^{n}$ is the state vector

Let the system be controlled by a stabilizing feedback controller given by:

$$
\Sigma_{C}:\{u=K y
$$

The coprime factorization of the nominal system $G_{y u}$ from (1) and the stabilizing controller $K$ from (3) are given by:

$$
\begin{array}{llrl}
G_{y u} & =N M^{-1}=\tilde{M}^{-1} \tilde{N}, & N, M, \tilde{N}, \tilde{M} \in \mathcal{R} \mathcal{H}_{\infty} \\
K & =U V^{-1}=\tilde{V}^{-1} \tilde{U}, & U, V, \tilde{U}, \tilde{V} \in \mathcal{R H}_{\infty}
\end{array}
$$

where the eight matrices in (4) must satisfy the double Bezout equation given by, see [23]:

$$
\begin{aligned}
\left(\begin{array}{ll}
I & 0 \\
0 & I
\end{array}\right) & =\left(\begin{array}{cc}
\tilde{V} & -\tilde{U} \\
-\tilde{N} & \tilde{M}
\end{array}\right)\left(\begin{array}{cc}
M & U \\
N & V
\end{array}\right) \\
& =\left(\begin{array}{cc}
M & U \\
N & V
\end{array}\right)\left(\begin{array}{cc}
\tilde{V} & -\tilde{U} \\
-\tilde{N} & \tilde{M}
\end{array}\right)
\end{aligned}
$$

Based on the above coprime factorization, a parameterization of all controllers that stabilize the system in terms of a stable transfer function $Q$, i.e. all stabilizing controllers are given by using a right factored form [18]:

$$
K(Q)=(U+M Q)(V+N Q)^{-1}, Q \in \mathcal{R} \mathcal{H}_{\infty}
$$

or by using a left factored form:

$$
K(Q)=(\tilde{V}+Q \tilde{N})^{-1}(\tilde{U}+Q \tilde{M}), Q \in \mathcal{R} \mathcal{H}_{\infty}
$$

Using the Bezout equation, the controller given either by (6) or by (7) can be realized as an LFT in the parameter $Q$ :

$$
K(Q)=\mathcal{F}_{l}\left(\left(\begin{array}{cc}
U V^{-1} & \tilde{V}^{-1} \\
V^{-1} & -V^{-1} N
\end{array}\right), Q\right)=\mathcal{F}_{l}\left(J_{K}, Q\right)
$$

where $\mathcal{F}_{l}(\cdot, \cdot)$ is a lower linear fractional transformation (LFT), [16].

The Youla parameterization for the right factored form in (6) is shown in Fig. 1 and for the left factored form in (7) is shown in Fig. 2.

Based on the Youla parameterization shown above, it is possible to describe the closed-loop matrix transfer function from external input $w$ to external output $z$ as an LFT function of the Youla matrix transfer function $Q$. The closed-loop matrix transfer function $T_{c l}(Q)$ is given by:

$$
\begin{aligned}
T_{c l}(Q) & =G_{z w}+G_{z u} M \tilde{U} G_{y w}+G_{z u} M Q \tilde{M} G_{y w} \\
& =T_{z w}+T_{z \eta} Q T_{\varepsilon w}
\end{aligned}
$$

(9) shows the well-known result that $T_{c l}(Q)$ is an affine matrix function of $Q$.

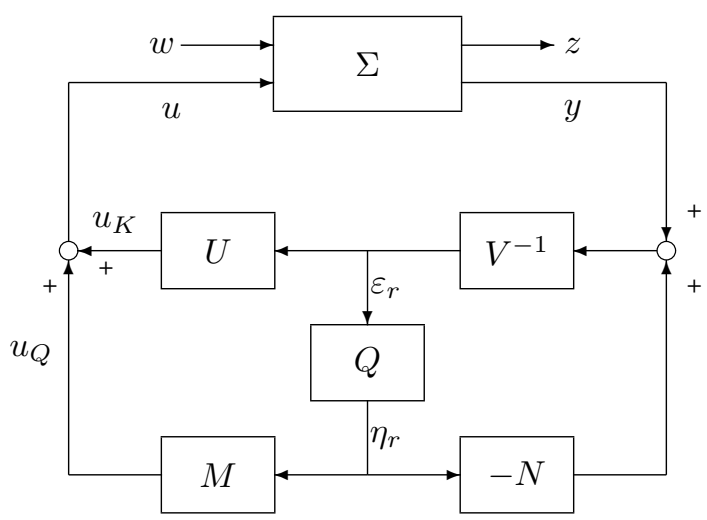

Fig. 1. The Youla parameterization of all stabilizing controllers $K(Q)$ based on (6) for a given system $\Sigma$.

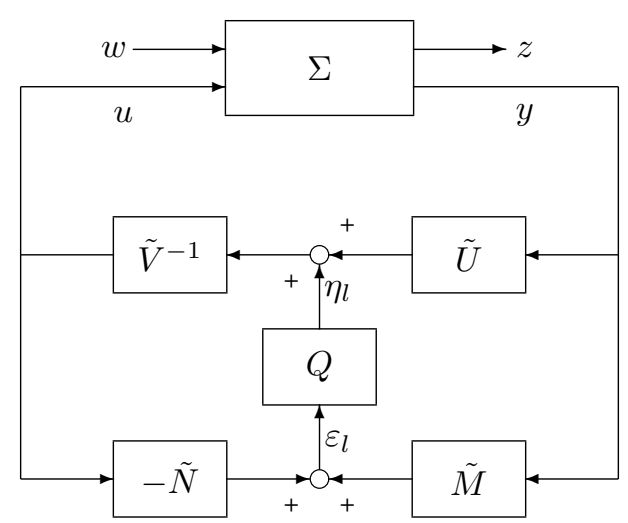

Fig. 2. The Youla parameterization of all stabilizing controllers $K(Q)$ based on (7) for a given system $\Sigma$.

\section{INPUT-OUTPUT IMPLEMENTATION}

The affine matrix transfer function given by (9) shows that the affine part does not depend directly on the applied feedback controller. However, as shown in Fig. 1 and Fig. 2 , the implementation of the architectures requires access to an internal loop point in nominal feedback controller.

This can be satisfied by using an implementation in terms of either a matrix transfer function or the coprime factors for the controller. If it is not possible to get internal signals in the controller, it is not possible to implement the Youla parameterization in the form described above.

The first step in reformulation of the controller parameterization is to consider the two implementations of the Youla controller architecture shown in Fig. 1 and Fig. 2. From Fig. 1 , the matrix transfer function from $\eta_{r}$ to $z$ is given by

$$
T_{z \eta}=G_{z u} M
$$

$T_{z \eta}$ is independent of the feedback controller. $T_{z \eta}$ can be calculate without using the feedback controller. Using Fig. $1, u$ is given by:

$u=M \eta_{r}+U V^{-1} y-U V^{-1} N \eta_{r}=K y+(M-K N) \eta_{r}$ 
Using the coprime factorization of the controller together with the Bezout equation, (11) can then be rewritten into:

$$
u=K y+\tilde{V}^{-1}(\tilde{V} M-\tilde{U} N) \eta_{r}=K y+\tilde{V}^{-1} \eta_{r}
$$

(12) show that the control input $u$ from Fig. 1 is exact the same as in Fig. 2. This show that instead of adding the $\eta$ vector internally into the controller, it can be added to the input and output from the controller.

It is also easy to show the other way around. Using the Bezout equation in (5), we have:

$$
\tilde{V}^{-1}=M-K N
$$

Using $\tilde{V}^{-1}$ from (13) in (12) gives (11).

Now, let's consider Fig. 2 with respect to the matrix transfer function from $w$ to $\varepsilon_{l}$. $T_{\varepsilon w}$ is given by:

$$
T_{\varepsilon w}=\tilde{M} G_{y w}
$$

Again, $T_{\varepsilon w}$ is independent of the feedback controller and $T_{\varepsilon w}$ can be calculate without using the feedback controller. It is also here possible to show that the two vectors $\varepsilon_{l}$ and $\varepsilon_{r}$ is identical. From Fig. $2 \varepsilon_{l}$ is given by:

$$
\varepsilon_{l}=\tilde{M} y-\tilde{N} u=(\tilde{M}-\tilde{N} K) y-\tilde{N} \tilde{V}^{-1} \eta_{l}
$$

Using the coprime factorization of the controller together with the Bezout equation, (15) can then be rewritten into:

$$
\varepsilon_{l}=(\tilde{M} V-\tilde{N} U) V^{-1} y-\tilde{N} \tilde{V}^{-1} \eta_{l}=V^{-1}\left(y-N \eta_{l}\right)
$$

showing that $\varepsilon_{l}=\varepsilon_{r}$.

Based on these calculations, the two equivalent controller architectures for the Youla parameterization shown in Fig. 1 and Fig. 2 can now be reformulated. The two reformulated controller architectures are shown in Fig. 3 and in Fig. 4, respectively.

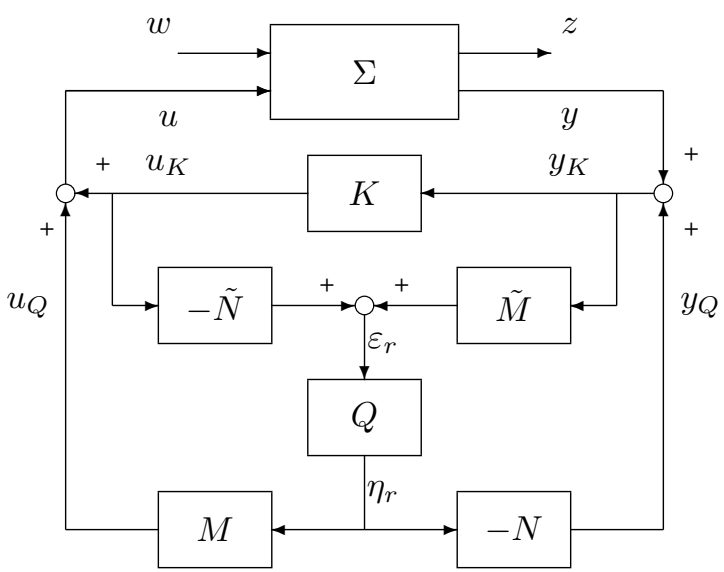

Fig. 3. A reformulation of the Youla controller architecture shown in Fig. 1 gives this controller implementation only based on inputs and outputs from the nominal controller.

The two controller architectures shown in Fig. 3 and in Fig. 4, respectively, is identical. They gives the same two outputs $u$ and $\varepsilon$. It is easy to see that the control vector $u$ is given by (11) or (12) for both architectures. Note that

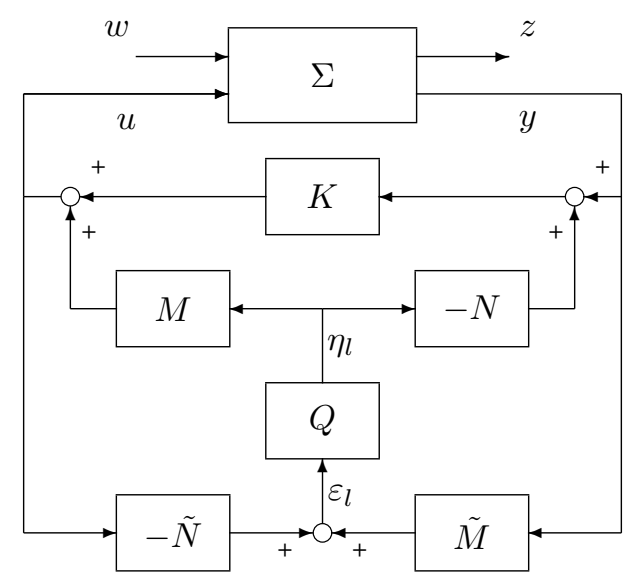

Fig. 4. A reformulation of the Youla controller architecture shown in Fig. 2 gives this controller implementation only based on inputs and outputs from the nominal controller.

the control vector given by (11) is in line with the LFT description of $K(Q)$ given by (8).

It is important to point out that there is no pole-zero cancellation in RHP (right half plane) in connection with the calculation of $M-K N$ in (11) as well as for the calculation of $\tilde{M}-\tilde{N} K$ in (15). RHP zeros in $G_{y u}$ will occur in the coprime factors $N$ or $\tilde{N}$. Assuming that the closed-loop system given by $\left(G_{y u}, K\right)$ satisfy internal stability guarantee that there will be no pole-zero cancellation between $N$ or $\tilde{N}$ and the feedback controller $K$. Further, it is easy to calculate the matrix transfer function from the input $\eta$ to the control input vector $u$ (with $Q=0$ ). This gives:

$$
\begin{aligned}
u & =\left(I-K G_{y u}\right)^{-1} M \eta_{l}-\left(I-K G_{y u}\right)^{-1} K N \eta_{l} \\
& =\left(\tilde{V}-\tilde{U} G_{y u}\right)^{-1} \tilde{V} M \eta_{l}-\left(\tilde{V}-\tilde{U} G_{y u}\right)^{-1} \tilde{U} N \eta_{l} \\
& =M \tilde{V} M \eta_{l}-M \tilde{U} N \eta_{l} \\
& =M \eta_{l}
\end{aligned}
$$

Let's consider the parameterization part of the controller in Fig. 4. This is shown in Fig. 5.

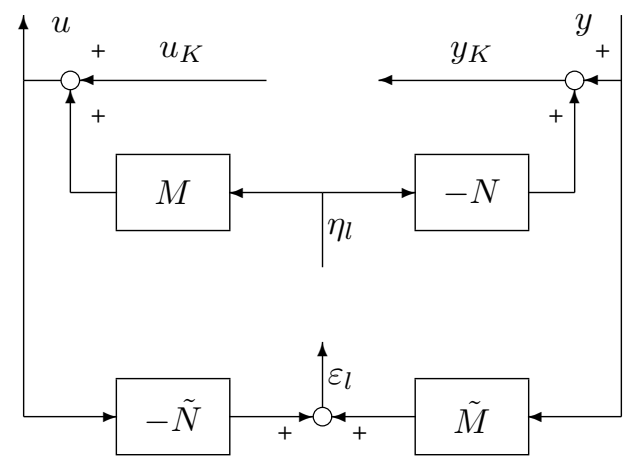

Fig. 5. The parameterization part of the Youla controller architecture shown in Fig. 4.

The input-output description of the system shown in Fig. 
5 is given by:

$$
\left(\begin{array}{c}
u \\
y_{K} \\
\varepsilon_{l}
\end{array}\right)=\left(\begin{array}{ccc}
I & 0 & M \\
0 & I & -N \\
-\tilde{N} & \tilde{M} & -\tilde{N} M
\end{array}\right)\left(\begin{array}{c}
u_{K} \\
y \\
\eta_{l}
\end{array}\right)
$$

The controller in Fig. 3 have the same input-output description.

It can also be mentioned that the anti-windup controller architecture described in [11] is based on the same inputoutput implementation of the Youla architecture as described in this paper.

It is also possible to give a state-space description of the input-output description given by (18). For this, state-space representations of the coprime factors for $G_{y u}$ are needed. Let a state feedback matrix $F$ be selected such that $A+$ $B_{u} F$ is stable and let an observer matrix $L$ be selected such that $A+L C_{y}$ is stable. A state-space representation of the coprime factors for $G_{y u}$ can then be given by, [18]:

$$
\begin{aligned}
&\left(\begin{array}{c}
M \\
N
\end{array}\right)=\left(\begin{array}{c|c}
A+B_{u} F & B_{u} \\
\hline F & I \\
C_{y}+D_{y u} F & D_{y u}
\end{array}\right) \\
&\left(\begin{array}{cc}
-\tilde{N} & \tilde{M}
\end{array}\right)=\left(\begin{array}{c|cc}
A+L C_{y} & -\left(B_{u}+L D_{y u}\right) & L \\
\hline C_{y} & -D_{y u} & I
\end{array}\right)
\end{aligned}
$$

(18) is then given by:

$$
\left(\begin{array}{c}
\dot{x}_{F} \\
\dot{x}_{L} \\
u \\
y_{k} \\
\varepsilon_{L}
\end{array}\right)=\left(\begin{array}{cc|ccc}
A_{F} & 0 & 0 & 0 & B_{u} \\
B_{L} F & A_{L} & -B_{L} & L & -B_{L} \\
\hline F & 0 & I & 0 & I \\
-C_{F} & 0 & 0 & I & -D_{y u} \\
-D_{y u} F & C_{y} & -D_{y u} & I & -D_{y u}
\end{array}\right)\left(\begin{array}{c}
x_{F} \\
x_{L} \\
u_{K} \\
y \\
\eta_{L}
\end{array}\right)
$$

where $A_{F}=A+B_{u} F, A_{L}=A+L C_{y}, B_{L}=B_{u}+L D_{y u}$ and $C_{F}=C_{y}+D_{y u} F$.

\section{EXAMPLE}

Consider $G$ and $K$ are given by:

$$
\begin{aligned}
G & =\frac{1}{s-1} \\
K & =-k \frac{\tau s+1}{\tau s}
\end{aligned}
$$

where $k$ is the controller gain and $\tau$ is the integration time constant in the PI-controller. It is necessary to required that $k>1$ for having closed-loop stability.

A coprime factorization of $G$ is given by:

$$
N=\frac{1}{s+1}, M=\frac{s-1}{s+1}
$$

Further, a coprime factorization of $K$ satisfying the Bezout equation is given by:

$$
\begin{aligned}
V & =\frac{\tau s(s+1)}{\tau s^{2}+\tau(k-1) s+k} \\
U & =-k \frac{(\tau s+1)(s+1)}{\tau s^{2}+\tau(k-1) s+k}
\end{aligned}
$$

Let's consider a reference tracking design. Let the external input $w$ be the reference input, i.e. $w=r$ and let the external output be the tracking error $e$, i.e. $z=e$. The general system $\Sigma$ in (1) is then given by, [16]:

$$
\Sigma:\left\{\left(\begin{array}{l}
z \\
y
\end{array}\right)=\left(\begin{array}{l}
1-\frac{1}{s-1} \\
1-\frac{1}{s-1}
\end{array}\right)\left(\begin{array}{l}
w \\
u
\end{array}\right)\right.
$$

The general setup for this system is shown in Fig. 6. It is here important to note that the Youla part of the controller architecture is an add-on to the existing nominal controller. Fig. 6 shows clearly how it is possible to implement the Youla architecture without using information about the nominal controller.

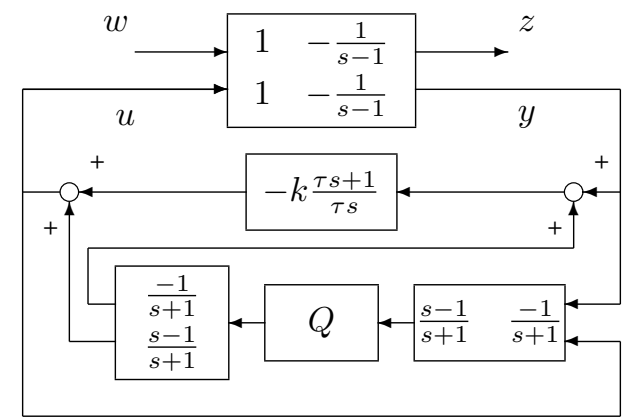

Fig. 6. The controller architecture for the system setup.

Using (9), the closed-loop transfer function $T_{z w}$ from $r$ to $e$ in Fig. 6 is then given by:

$$
\begin{aligned}
T_{z w}(Q) & =\frac{1}{1+G K}+G M Q M \\
& =S+N Q M
\end{aligned}
$$

where $S$ is the sensitivity transfer function. Using the coprime factorization of $G$ and $K,(26)$ can be rewritten into:

$$
T_{z w}(Q)=(V+Q N) M
$$

Applying the PI-controller given by (22), the closed-loop transfer function is given by:

$$
T_{z w}(Q)=\frac{\tau s(s-1)}{\left(\tau s^{2}+\tau(k-1) s+k\right)(s+1)}+Q \frac{s-1}{(s+1)^{2}}
$$

or

$$
T_{z w}(Q)=\left(\frac{s(s+1)}{s^{2}+(k-1) s+k / \tau}+Q \frac{1}{s+1}\right) \frac{s-1}{s+1}
$$

In the first design, assume that the nominal PI controller is known. Let the gains of the PI controller be given by:

$$
\Sigma_{C_{1}}: k_{1}=2.0 \quad \tau_{1}=0.25
$$

Now, consider a decoupling design of $Q$. From (26) we have that $Q$ given by

$$
\begin{aligned}
Q & =-\frac{\tau s(s+1)}{\tau s^{2}+\tau(k-1) s+k} \frac{s+1}{\alpha s+1} \\
& =-\frac{s(s+1)}{s^{2}+s+8} \frac{s+1}{\alpha s+1}
\end{aligned}
$$

will give an approximative decoupling when $\alpha$ is selected as small positive constant, i.e. a design where the transfer function $T_{z w}(Q)$ reduced. The result of the design is shown in Fig. 7 for different values of $\alpha$.

It is clear from Fig. 7 that reducing $\alpha$ gives a $Q$ that will given better reference tracking, i.e. the tracking error given by $T_{z w}$ goes to zero faster.

In the second design, $Q$ will be designed such that a specified closed-loop system $T_{z w}$ is obtained. Further, it is 


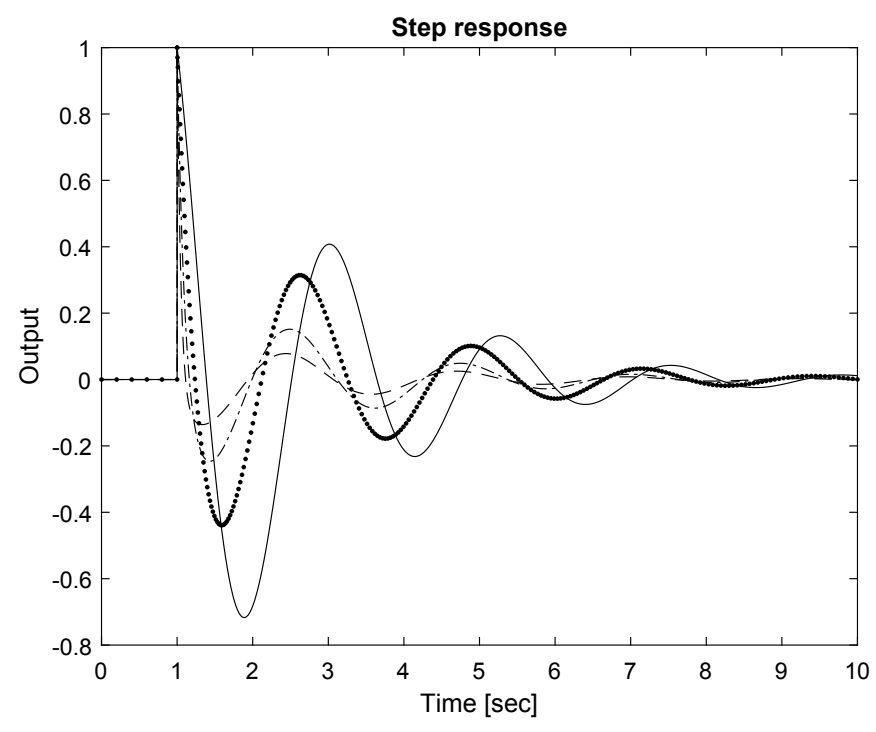

Fig. 7. Simulation of the closed-loop system with the PI-controller given by $\Sigma_{C_{1}}$ (solid line) as the nominal controller $(Q=0)$. The three other simulation are when the Youla matrix transfer function $Q$ given by (31) is applied for $\alpha=0.25$ (dotted line), 0.10 (dashed-dotted line) and 0.05 (dashed line).

assumed that the parameters for the PI-controller given in (30) is unknown. The nominal closed-loop transfer function is given by:

$$
T_{z w}=\frac{s(s-1)}{s^{2}+a s+b}
$$

where $a$ and $b$ are unknown positive constants. Further, let the desired closed-loop transfer function be given by:

$$
T_{z w, \text { desired }}=\frac{s(s-1)}{s^{2}+6.5 s+7.5 / 0.45}
$$

This closed-loop system can be obtained by the following parameters for the PI-controller:

$$
\Sigma_{C_{2}}: k_{2}=7.5 \quad \tau_{2}=0.45
$$

The simulation of the closed-loop system with the two controllers is shown in Fig. 8. It is clear from Fig. 8 that using $\Sigma_{C_{1}}$ as the feedback controller gives a poor performance whereas $\Sigma_{C_{2}}$ gives a much better performance. $\Sigma_{C_{1}}$ will in the following be used as the nominal feedback controller where the

Based on the closed-loop transfer function $T_{z w}(Q)$ given by (29) together with the desired closed-loop transfer function $T_{z w \text {,desired }}$ given by (33) gives directly $Q$ as:

$$
\begin{aligned}
Q & =s(s+1)^{2}\left(\frac{1}{s^{2}+6.5 s+7.5 / 0.45}-\frac{1}{s^{2}+a s+b}\right) \\
& =s(s+1)^{2} \frac{(a-6.5) s+(b-7.5 / 0.45)}{\left(s^{2}+6.5 s+7.5 / 0.45\right)\left(s^{2}+a s+b\right)}
\end{aligned}
$$

The two parameters $a, b$ can be estimated directly from the nominal closed-loop system. Instead, the optimization of the two parameters can also be done directly using $Q$ given above. Standard methods for optimization of the parameters can be applied. Here, we will only show the effect on the response of the closed-loop system using different values for the two parameters in $Q$. The step responses are shown in

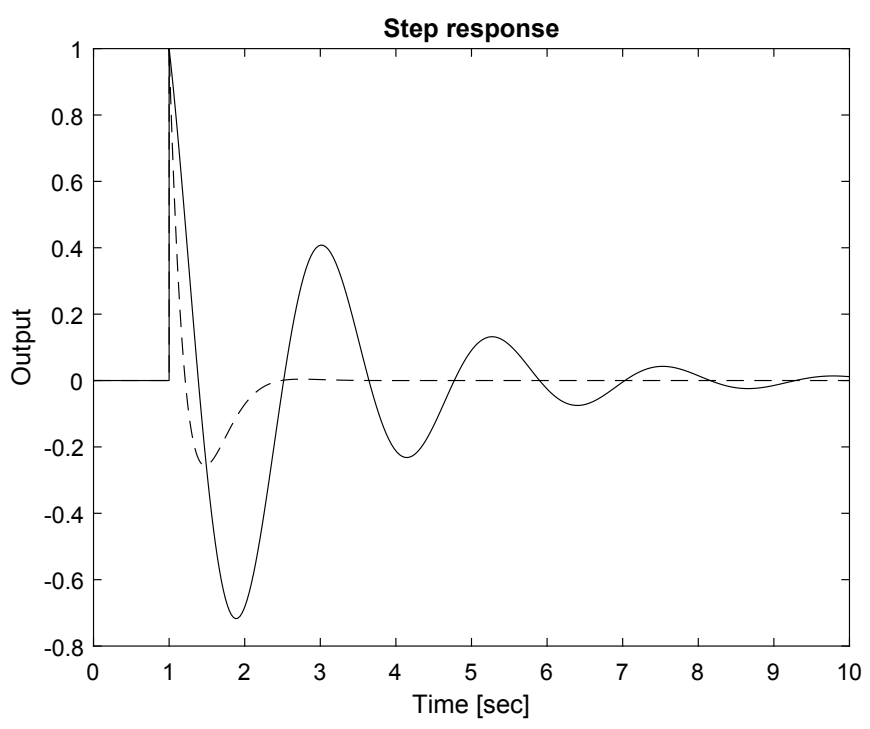

Fig. 8. Simulation of the closed-loop system with the PI-controller given by $\Sigma_{C_{1}}$ (solid line) and with $\Sigma_{C_{2}}$ (dashed line).

Fig. 9 and 10. In both figures, the solid line is for the step response with $a=1$ and $b=8$, i.e. the desired response.

Fig. 9 show the step responses for change in $a$ with $b=8$. The values for $a$ are: $0.25,0.50,0.75,1.00,1.25$ and 1.50 . Fig. 10 show the step responses for change in $b$ with $a=1$. The values for $b$ are: 6.0, 7.0, 8.0, 9.0 and 10.0.

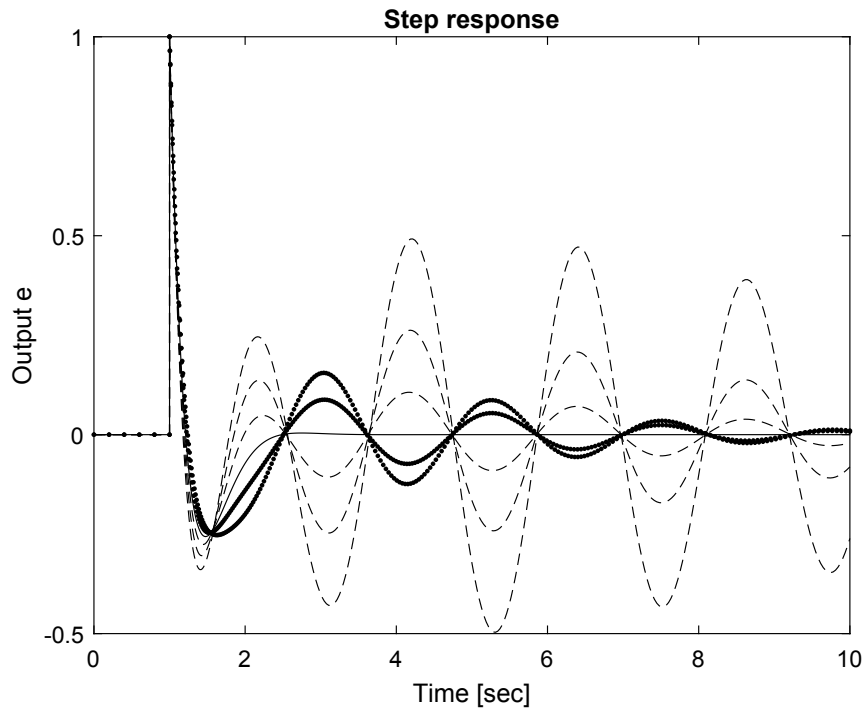

Fig. 9. Simulation of the closed-loop system with the PI-controller given by $\Sigma_{C_{1}}$ as the nominal controller. The step responses are shown for $b=8$ and with $a=0.25,0.50,0.75$ (dashed lines), $a=1.0$ (solid line) and for $a=1.25,1.50$ (dotted line).

An estimation or learning-based method needs to be applied for the optimization of the two parameters $a$ and $b$ as pointed out above.

At last, it should be mentioned that knowing the nominal controller, the change of the controller from $\Sigma_{C_{1}}$ to $\Sigma_{C_{2}}$ by using $Q, Q$ will be given by, [7], [14]:

$$
Q=U_{2} V-V_{2} U
$$




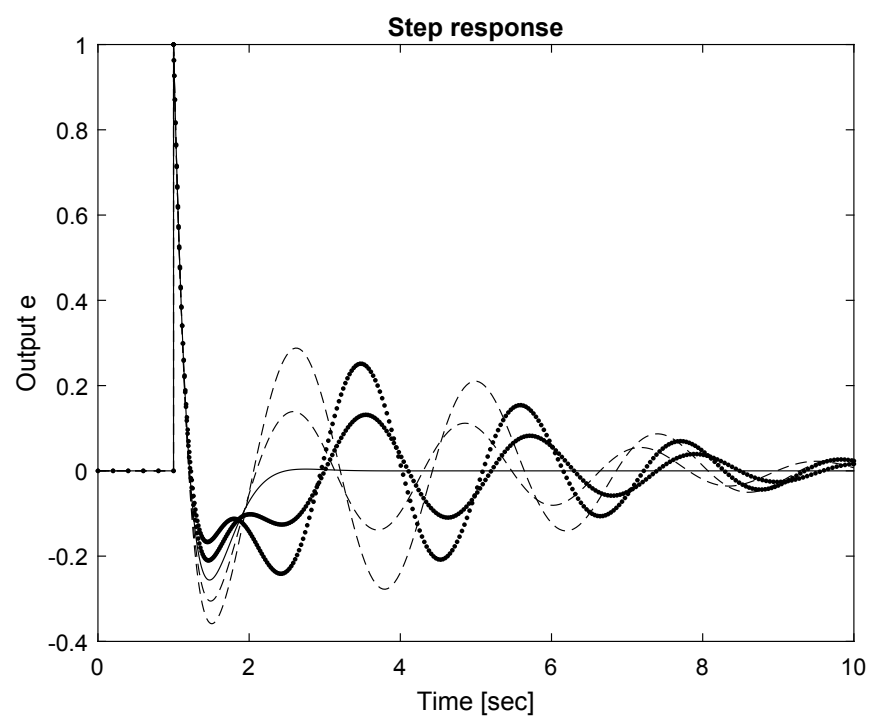

Fig. 10. Simulation of the closed-loop system with the PI-controller given by $\Sigma_{C_{1}}$ as the nominal controller. The step responses are shown for $a=1$ and with $b=6.0,7.0$ (dashed lines), $b=8.0$ (solid line) and for $b=$ 9.0, 10.0 (dotted line).

where $U_{2}$ and $V_{2}$ in (36) are the coprime factors for the new controller selected such that the Bezout equation is satisfied. It should be pointed out that this is a special case of the more general case considered in [7], [14]. With the parameters given for the two PI-controllers, $Q$ is given by:

$$
Q=-\frac{5.50 s^{4}+19.67 s^{3}+22.83 s^{2}+8.67 s}{s^{4}+7.50 s^{3}+31,17 s^{2}+68.67 s+133.33}
$$

If we use $a=1$ and $b=8$ in (35), we get the same $Q$ as in (37).

\section{CONCLUSion}

The Youla controller architecture has been considered in this paper. It has been shown that it is possible to reformulate the Youla architecture such that the parameterization part can be implemented around the nominal feedback controller without using internal signals from this. It is only required a left and right coprime factorization of the nominal system. This new Youla architecture will allow us to optimize closedloop performance on the existing closed-loop system without using an internal controller connection.

\section{REFERENCES}

[1] B.D.O. Anderson. From Youla-Kucera to identification, adaptive and nonlinear control. Automatica, 34(12):1485-1506, 1998.

[2] S.P. Boyd and C.H. Barratt. Linear controller design - limits of performance. Prentice Hall, 1991.

[3] M.A. Dahleh and I.J. Diaz-Bobillo. Control of Uncertain systems. Prentice Hall, 1995.

[4] L. Furieri, Y. Zheng, A. Papachristodoulou, and M. Kamgarpour. An inputâĂŞoutput parametrization of stabilizing controllers: Amidst youla and system level synthesis. IEEE Control Systems Letters, 3(4):1014-1019, 2019.

[5] V. Kucera. Stability of discrete linear feedback systems. In Proceedings of the 6th IFAC World Congress, Boston, MA, 1975. Paper 44.1.

[6] H. Luo, H. Zhao, S.X. Ding, S. Yin, H. Gao, and X. Yang. Scalability of feedback control systems for plug-and-play control. In Proceedings of the 20th IFAC World Congress, volume 50(1), pages 7529-7534, Toulouse, France, 2017.
[7] J.B. Moore, K. Glover, and A. Telford. All stabilizing controllers as frequency-shaped state estimate feedback. IEEE Transactions on Automatic Control, 35:203-208, 1990.

[8] M. Morari and E. Zafirious. Robust Process Control. Prentice-Hall, Englewood Cliffs, New Jersey, 1989.

[9] C.N. Nett, C.A. Jacobson, and M.J. Balas. A connection between state space and doubly coprime fractional representations. IEEE Transactions on Automatic Control, 29(9):831-832, 1984.

[10] H.H. Niemann. A setup for active fault diagnosis. IEEE Transactions on Automatic Control, 51(9):1572-1578, 2006.

[11] H.H. Niemann. A controller architecture with anti-windup. IEEE Control Systems Letters, 4(1):139-144, 2020.

[12] H.H. Niemann, R. Miklos, and N.K. Poulsen. Grey box identification in closed-loop. In Proceedings of the American Control Conference, pages 4990-4995, Philadelphia, PA, USA, 2019.

[13] H.H. Niemann and J. Stoustrup. An architecture for fault tolerant controllers. International Journal of Control, 78(14):1091-1110, 2005.

[14] H.H. Niemann, J. Stoustrup, and R.B. Abrahamsen. Switching between multivariable controllers. Optimal Control - Application and Methods, pages 51-66, 2004.

[15] M. Rotkowitz. Information structures preserved under nonlinear timevarying feedback. In Proceedings of the American Control Conference, pages 418-423, Minneapolis, Minnesota, USA, 2006.

[16] S. Skogestad and I. Postlethwaite. Multivariable feedback control: Analysis and Design. Wiley, 2005.

[17] J. Stoustrup. Plug \& play control: Control technology towards new challenges. European Journal of Control, 15(3-4):311-330, 2009.

[18] T.T. Tay, I.M.Y. Mareels, and J.B. Moore. High performance control. Birkhäuser, 1997.

[19] Y. Wang, N. Matni, and J. C. Doyle. A system-level approach to controller synthesis. IEEE Transactions on Automatic Control, 64(10):4079-4093, 2019

[20] D.C. Youla, J.J. Bongiorno, and H.A. Jabr. Modern Wiener-Hopf design of optimal controllers - Part I The single-input-output case. IEEE Transactions on Automatic Control, 21(1):3-13, 1976.

[21] D.C. Youla, H.A. Jabr, and J.J. Bongiorno. Modern Wiener-Hopf design of optimal controllers - Part II: The multivariable case. IEEE Transactions on Automatic Control, 21(3):319-338, 1976.

[22] Y. Zheng, L. Furieri, A. Papachristodoulou, N. Li, and M. Kamgarpour. On the equivalence of youla, system-level and input-output parameterizations. IEEE Transactions on Automatic Control, 66(1):413-420, 2021

[23] K. Zhou, J.C. Doyle, and K. Glover. Robust and optimal control. Prentice Hall, 1995. 